\title{
An Experimental Evidence of Gasoline Ionization by a Magnetic Field
}

\author{
Raul Guerrero Torres ${ }^{1}$, Mehrab Mehrvar ${ }^{2}$ \\ ${ }^{1}$ Faculty of Engineering, University of Cartagena \\ Cartagena de Indias, Colombia \\ rguerrerotores1@unicartagena.edu.co \\ ${ }^{2}$ Department of Chemical Engineering, Ryerson University \\ 350 Victoria Street, Toronto, ON, Canada M5B 2K3 \\ mmehrvar@ryerson.ca
}

\begin{abstract}
The objective of this paper is to study an experimental evidence of gasoline ionization by a magnetic field. Results could be understood as an experimental evidence of what has been concluded, but not scientifically explained completely, since almost eight decades from underlying theory on the action of magnetic fields on liquids, confirmed empirically in the last three decades by better performance of mobile sources using efficient fabricated magnetic fuel optimizers; Ethos MAX Power Super Fuel in England and Fuel Optimizer in Argentina, to mention only two efficient magnetic devices, as well as many magnetic fluid conditioners patented in the last three decades in USA, and the results for Carbon monoxide (CO) and hydrocarbons (HC) emissions obtained from gas analyzers of two ADC (Automotive Diagnostic Centres) in the city of Cartagena, Colombia, in 2008. The results are also experimental evidence of the influence of hydraulic changes in flow conditions (section enlargement) before the action of the magnetic field, in the whole process of optimizing the fuel combustion. Moreover, the results show that the magnetic field action on the fuel is more efficient when magnets are within the conduction hose than that when placed externally to it. An experimental set up was used for measuring DC current through standard gasoline RON 87 under five different conditions; no magnets with or without altered flow conditions, with 2400 gauss neodymium magnets within the conduction hose with or without altered flow conditions and with neodymium magnets placed externally on the conduction hose. Successive values of 50nA, $320 \mu \mathrm{A}, 780 \mu \mathrm{A}, 2.7 \mathrm{~mA}$ and $4.8 \mathrm{~mA}$ were measured, suggesting ions increase, and consequently electrical resistivity decrease. Four apparent electrical resistivities (and apparent electrical conductivities) were calculated and important different grades of magnitude were found.
\end{abstract}

Keywords: Ionization, DC current, Neodymium Magnets, RON 87, Apparent Resistivity, Magnetic Fuel Optimizer.

\section{Introduction}

The use of powerful magnetic fields to improve the combustion efficiency in IC engines has been studied for a while. The use of magnetic fields to improve the performance of combustion was reported as early as the 1940s. The U.S. Air Force used a device on their Mustang aircraft that allowed a greater range and a better performance from poor quality fuels. This was proved successfully where it was subsequently used by the Royal Air Force on Spitfire and Hurricane aircrafts. The devices used back then were very heavy and cumbersome, as they had to use electric current to produce the required strength of magnetic field. But today, with the advent of new neodymium super magnets, even more powerful magnetic fields can be generated by units little larger than a matchbox.

Bloch and Purcell, who were jointly awarded in 1952 by studies on the action of magnetic fields on liquids and solids had studied the induction of nuclear spin in the atoms by the action of a magnetic field ${ }^{1}$, giving a scientific fundamentals to explain the empirical evidence that the use of powerful magnetic fields optimize fuel combustion to the extent that when a very powerful magnetic field is applied directly to a fuel supply line, it conditions the fuel in such a way that it combines more readily with the oxygen in the air and thus, burns more completely when combusted. Consequently, this enhanced burn produces more power from the same given volume of fuel and exhausts far less pollutants, as the major part of the exhaust pollution consists of the un-burnt fuel particulates. Today this can be verified easily and quickly demonstrated using standard emissions testing equipment as used in an authorized Automotive Diagnostic Centre or laboratory that guarantees the quality of this service. 
In 1992, The Warren Springs Laboratories ${ }^{1}$, the UK Government DTI's own vehicle-testing department, were commissioned to carry out extensive trials on the benefits of magnetic fuel conditioning technology and were very surprised by the results. Their report confirmed an increase in power, an improved fuel economy and a reduction in harmful emissions. Summing up in their conclusions that "both series of tests showed consistent reductions in carbon dioxide, fuel consumption, and oxides of nitrogen, and when the test unit was removed the trend was reversed". In addition, the power increase observed in the first series of the tests was sustained throughout the second series of the tests and marginally increased to $11 \%$.

Since the discovery of Bloch and Purcell to date, many types of magnetic devices have been built to optimize combustion and/or solve specific problems related to its efficiency or the fuel properties and/or working conditions. There are three specific efficient- fuels, available to buy, among them, as follows:

- Ethos MAX Power Super Fuel²; Built in England. Available to buy at $70 £$

- Fuel Optimizer ${ }^{3}$; Built in Argentina. Available to buy at \$34 US/unit dollars for vehicles up to 2500cc

- High Magnetic Fuel Saver for Energy and Performance ; Built in China and for sell at \$119 US dollars/unit

These three magnetic fuel optimizers of the highest level of the state of the art magnetic devices are generally installed externally, in the fuel lines, except in case when the pipe is lined with a wire mesh. In that case, the device is assembled on a copper, aluminum or steel pipe. The fuel supply pipeline is cut and the ends of the device are fitted into the lined fuel supply pipeline with two clamps. These devices are not always efficient enough for ionizing the fuel because they do not provide adequate shielding, necessary to counteract the action of random fields that can affect adversely ionization of the fuel. Furthermore, actions such as the magnetic field of the earth in addition to unfavorable external conditions may result in a deficient fuel ionization because the neodymium magnets action would be considerably reduced by the external conditions and in some cases, the device would not work at all. Today, as revealed by an internet search, no-less than 50 magnetic fuel savers of different kinds built in different countries are available to buy at a wide scale of prices ranging from $\$ 1.70$ to $\$ 119$ US dollars.

Although there are many magnetic devices to optimize combustion in IC vehicles patented in Europe and US since the fifties', built mostly by Chinese inventors, as can be proved by a search in the US Patent Office web page and ESPACENET, web page of the Europe Patent Office, only those related somehow with the purpose of the experiment are shown below, emphasizing only in a device whose characteristics are strongly related with one of the objectives of the experiment and are integral part of it, quantitative study of devices assembling fluid splitters for changing the fuel flow conditions and magnets. Therefore, we emphasize in the fuel saver device patented in USA under number 5,673,674. However, there are older and newer devices, whose details may be reviewed in USPTO and related as follows:

- Means for imposing Electric and Magnetic Fields On Flowing Fluids; US Patent Number 3,349,354

- Apparatus for magnetically treating fluids; US Patent Number 5,500,121

- Automobile fuel saver; US Patent Number 7,377,269

- Device for enhancing fuel efficiency of internal combustion engines; US Patent Number 7,412,974

- Fuel activation apparatus using magnetic body; US Patent Number 5,329,911

- Magnetic device for treating hydrocarbon fuels; US Patent Number 4,372,852

- Magnetic fluid conditioner; US Patent Number 4,995,425

- Magnetic fuel enhancer; US Patent Number 5,943,998

- Method and apparatus for magnetically treating fluids; US Patent Number 4,519,919

- Process and Apparatus for Effecting Efficient Combustion; US Patent Number 3830621

Fuel saver; US Patent Number 5,673,674

It is a fluid saving device including a fuel multidirectional device in a magnetic field. The multidirectional device includes a reservoir and a plurality of spirals surrounding the reservoir for passage of the fuel, in turbulent manner through a magnetic field. This turbulence splits the clusters of molecules of the fluid, ionize them and makes easier the magnetic field action to ionize the fuel because it breaks the cluster of molecules. 
This kind of devices making a pre-treatment to the fuel, before the magnetic field action results in more efficient devices as the minimizer presented in RETESE'17. PP Presentation ${ }^{5}$ and paper $^{6}$

One of the objectives of the experiments here reported was to quantify ionization of standard gasoline ROM 87, in the whole process of ionization of the fluid, due to the turbulence caused by a flow section enlargement of the fuel supply pipeline along $10 \mathrm{~cm}$, through the cross section of the minimizer device installed without magnets

The experiment was intended to answer questions as these:

How much the section enlargement influences the fuel ionization?

How much the section enlargement improves the whole fuel ionization?

How much the section enlargement favors the magnetic field action?

What is the rate between the enlargement ionization and the magnets ionization?

There are several studies and analysis on functioning of very specific magnetic devices most of them patented, as well as publications of papers in journals on the successful application of inferred conclusions from Bloch and Purcell to specific practical cases, few formal experimental studies on the behavior of fuels under the action of magnetic fields and very few, for not saying none experimental study found about variations of specific physical properties of fuels by the action of magnetic fields. A search in internet confirms all the statements, especially the last one.

This present experimental work follows the orientations of statement made by several leaders of the world in the VII International Convention on Environment and development held in Habana in July 9-12of 2009, in a few words: "Engineering alone can't solve completely the environmental problems of the world but working close together with science". It is time to start a close connection among Environmental Physics, Environmental Chemistry and engineering to solve the environmental problems of the world. This experimental work is a first step in the intention of applying scientific knowledge to study the behavior of specific physical properties of fuels under a magnetic field action, in this case, gasoline under the action of a magnetic field, in different conditions and in combination with the enlargement of the fuel flow section, through a simple experiment where the variables are not so many and its physical characterization in terms of the magnetic field as well as its control is not as complicated as could be in other more rigorous practical and technical instances but, scientifically, less formal and deep. The results of this kind of experimental works may be the support of some theoretical studies and a scientifically proof of practical application conjectures. The results of this work are very important not only as an experimental proof of the conditioning for better combustion of a fuel by a magnetic field action combined with flow changes but as an important information that may be correlated with the dimensioning and design of a magnetic fuel optimizer as the minimizer.

\section{Objectives}

The general objective was to prove the conjecture of the ionization of the gasoline by the magnetic field action and study its variations under different conditions; constant section without magnets, enlarged section of $15 \mathrm{~cm}$ long without magnets, constant section of the conduction hose with neodymium magnets of 2400 gauss placed externally on it, constant section with neodymium magnets of 2400 placed within the hose and enlarged section of $15 \mathrm{~cm}$ long till the couple of neodymium magnets of 2400 gauss within the conduction hose (Minimizer) The specific objectives were: to prove the conjecture of the important influence of section enlargement before the action of the magnetic field, in the whole process of optimizing the fuel combustion; and to prove the conjecture that the magnetic field action on the fuel is more efficient when magnets are within the conduction hose than placed externally to it.

\section{Experimental Setup and Procedure}

\subsection{Experimental Setup}

The first step was to sketch a model of the experimental setup to study the behavior of the gasoline under the magnetic field action and then assemble the setup elements to measure proper gasoline physical properties changing under the magnetic field action, based on the conjecture that a magnetic field should ionize the fuel, because when it exposes to the magnetic field a better combustion is achieved. A physical property of the gasoline closely correlated with ionization is the resistivity (or better, conductivity). To calculate the resistivity and/or conductivity of gasoline is necessary to measure the 
high resistance of gasoline, under each condition, by a special normalized procedure ${ }^{7}$ or to calculate it by measuring the potential difference (voltage) between two points and the correspondent DC current measured with a wide range digital multimeter. Though the multimeter used UT $50 \mathrm{~A}^{8}$ has not such wide range, it allowed to measure the currents through its inner resistance in parallel with the resistance of the gasoline and with information about the conductivity of gasoline ${ }^{9}$ made possible to reach the expected conclusions.

This model is sketched in Figure 1.

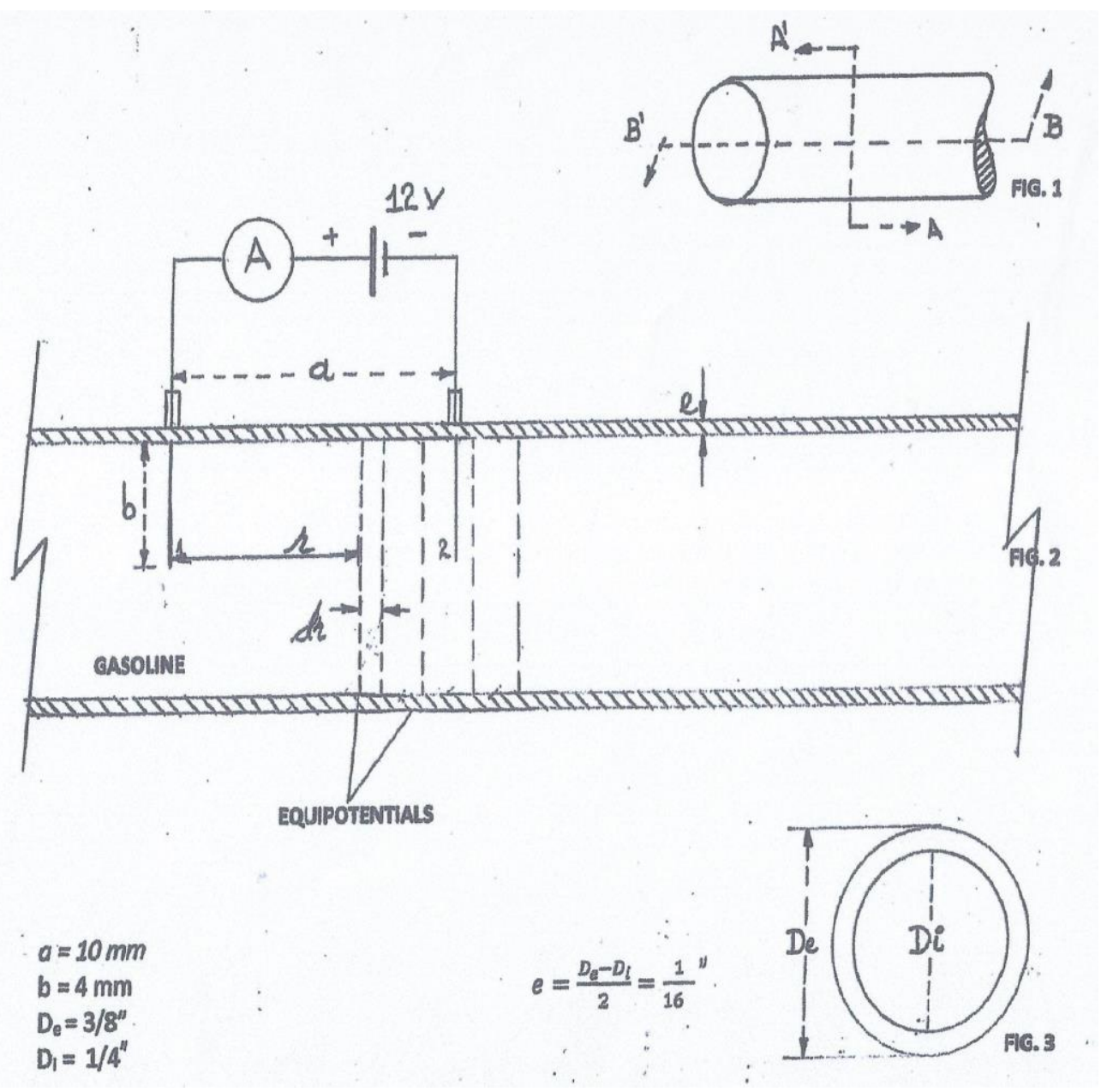

Fig. 1: Outline of the Experiment Set Up.

To obtain the measurements to characterize the gasoline behavior under the action of the magnetic field, a conduction system of five branches of polypropylene hoses 2 meter long with 0.25 inches in diameter controlled by valves, to set every condition, was used to carry the flow of gasoline by gravity from a plastic supply deposit of capacity 2.5 gal filled with 2.0 gal of gasoline to a second plastic recipient deposit of capacity 2.0 gal. A minimizer of gases emissions with 2400 gauss magnets, and then without them was coupled to the hose to set two different conditions, 2400 gauss magnets were coupled within the fuel supply hose to set other condition and two 2400 gauss magnets were installed on the fuel supply hose by means of a PVC tube piece coupled to it. A branch for fuel supply hose with non-additions was also used as shown in Figure 2 for sampling. 


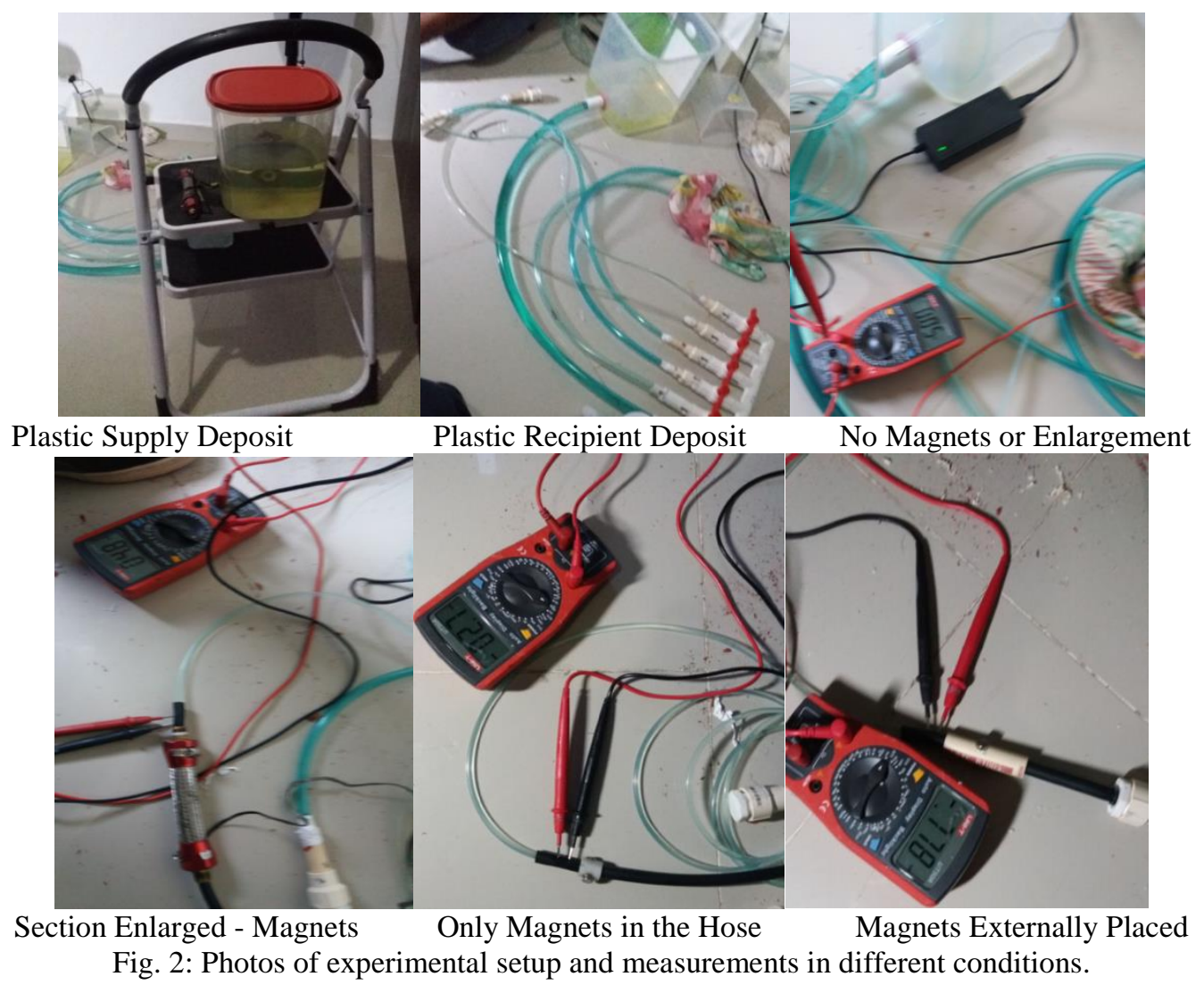

\subsection{Equipment and Materials}

1 Multimeter UT 50 A Red

1 AC-DC 12V Adapter, Model LE-1250, 100V-120V, 5A, f=60 Hz

1 Ubermann Digital Vernier Caliper

1 Electric Drill Dewalt 3/8

1 Fiberglass Tape Rule $10 \mathrm{~m}$ Long

1 Punch

1 Hacksaw Frame

1 Hacksaw Blade

1 Couple of 2400 gauss Neodymium Magnets

2 gallons

1 Polypropylene Hose $10 \mathrm{~m}$ long, diameter

1 Rubber hose piece $5 \mathrm{~cm}$ long diameter $1 / 4$

1 Plastic Deposit

1 Plastic Deposit

6 PVC Valves $1 / 2^{\prime \prime}$

6 PVC Adaptors $1 / 2 "$ to $1 / 4 "$

1.Teflon tape

$20 \mathrm{~g}$ of Hydraulic Sealant

\subsection{Procedure}

A previously assembled conduction system of 6 branches of polypropylene hoses 2 meter long with 0.25 inches in diameter' controlled by valves at their ends and connected both ends to PVC tubes $60 \mathrm{~cm}$ long and diameters $1 / 2$ " was placed on the floor. A plastic container with the capacity 10 liters filled with 7.5 liters of gasoline was placed at a height of $48 \mathrm{~cm}$ from the floor on an industrial ladder and connected to the system of five branches by a horizontal tube $10 \mathrm{~cm}$ and 
diameter $1 / 2$ "through a 900 coupling to a vertical PVC tube $55 \mathrm{~cm}$ height with 0.25 inches in diameter connected by a coupling to the 6 branches through one of the PVC tubes connected to them on the floor, with all the valves closed. Afterwards, an empty plastic container with the capacity 9.0 liters to receive the gasoline by gravity from the higher container was connected to the other end of the branches with all the valves closed. Finally, the valves connected to one branch were open and then the valves connected to the deposits to eliminate bubbles that could affect the flow and consequently the results of the experiment. It is important to mention that previous calculations were made to guarantee the flow of gasoline when making the measurements and prevent any gasoline spill to avoid accidents. The points of coupling were carefully sealed with a special hydraulic sealant.

\subsection{Measurements}

Due to the general and specific objectives, the experiment was limited to only one kind of gasoline; standard gasoline RON (Octane Number) 87, considered homogenous. The focus of this study was not in finding out what happens to ionization far from the magnets and this is why short branches of fuel supply hoses were used. The support of this decision is the following: we now empirically that combustion is improved by magnetic action from the results of CDA in Cartagena Colombia has shown. If the conjecture that this happens because magnetic action ionizes the fuel is correct, as theoretical knowledge may reveal, this ionization of the fuel should remain until the combustion chamber after traveling a distance whose length is close to the length of the branches of fuel supply hoses. Under these considerations, it was enough and practical, to take two points $1 \mathrm{~cm}$ apart within the fluid with approximately half of the diameter deep, connected at a potential difference of $12 \mathrm{v}$ and to measure currents in each of the 5 chosen cases; Unaltered hydraulic flow conditions with no magnets (natural flow of gasoline through a pipeline), enlarged section without magnets, magnets on the supply hose (no contact with the fuel), magnets within the fluid and magnets within the fluid and section enlarged (Minimizer) . Though is very important to do this experiment under wider conditions and more rigorous measurement control, it is important to point out that this small-scale experimental model has been very useful to achieve the expected conclusions. Perhaps a large scale and close to the reality model could make more difficult to reach the expected conclusions.

\section{Mathematical Models}

As the potential difference between the two points on the supply hose, chosen $1 \mathrm{~cm}$ apart, was constant and the cross section only was needed to measure currents and calculate resistances between the two points from Equation (1). Equation (2) gives the cross sectional area and Equation (3) is an arrangement to present the calculations as shown in Table 1. All cross sectional areas were equipotential; therefore, Equation (4) could be used to calculate the apparent resistivity $\rho$. Equation (5) expresses apparent conductivity $\sigma$ as the inverse of the apparent resistivity. Equation (6) expresses the total current, as the sum of the current in the fuel $I_{J}$ and the measured current through the resistance of the multimeter $i_{j}$ used to calculate apparent resistivities in each case. Equation (7) expresses the relation of a decreasing resistivity $\rho_{j}$ to $\rho_{0}=$ $10^{13} \Omega m$

in terms of the increasing relation of $I_{0}=3.8 \times 10^{-15} \mathrm{~A}$ to its correspondent current $I_{j}$.

$$
\begin{aligned}
& \left.R=\frac{V}{i} \text { (1), } A=\frac{\pi D^{2}}{4} \text { (2), } \frac{A}{l}=\frac{\pi D^{2}}{4 l} \text { (3), } \rho=R \frac{A}{l} \text { (4), } \sigma=\frac{1}{\rho}(5), I_{T_{j}}=I_{J}+i_{j} 6\right), \frac{\rho_{j}}{\rho_{0}}=\frac{I_{0}}{I_{j}} \text { (7) } \\
& \text { With } V=12 \mathrm{~V}, l=1 \mathrm{~cm}, D=\frac{1}{4} "=6.35 \times 10^{-3} \mathrm{~m} \text {, and the measured currents, table } 1 \text { was made. }
\end{aligned}
$$

Table 1: Results of measurements and calculations.

\begin{tabular}{|c|c|c|c|c|c|c|}
\hline \multicolumn{7}{|c|}{ CORRIENTES MEDIDAS RESISTIVIDADES Y CONDUCTIVIDADES DE LA GASOLINA EN EXPERIMENTO DE DICIEMBRE 22 DE 2017} \\
\hline CONDICIONES & VOLTAGE (V) & CURRENT i (A) & RESISTENCIA $\Omega(\mathrm{V} / \mathrm{i})$ & $\mathrm{A} / \mathrm{L}$ & RESISTIVIDAD $\Omega-\mathrm{m}$ & CONDUCTIVIDAD PS/m \\
\hline Unaltered flow conditions-no magnets & 12 & 5.0.E-08 & 2.4.E+08 & 3.2.E-03 & 7.7.E+05 & 1.30.E-06 \\
\hline Enlarged section-no magnets & 12 & 3.2.E-04 & 3.8.E+04 & 3.2.E-03 & 1.2.E+02 & 8.33.E-03 \\
\hline Magnets on the fuel supply hose & 12 & 7.8.E-04 & 1.5.E+04 & 3.2.E-03 & 4.9.E+01 & 2.03.E-02 \\
\hline Magnets in the fuel supply hose & 12 & 2.7.E-03 & 4.4.E+03 & 3.2.E-03 & 1.4.E+01 & 7.03.E-02 \\
\hline Minimizer Installed & 12 & 4.8.E-03 & $2.5 . \mathrm{E}+03$ & 3.2.E-03 & 8.0.E+00 & 1.25.E-01 \\
\hline
\end{tabular}

CORRIENTES MEDIDAS RESISTIVIDADES Y CONDUCTIVIDADES DE LA GASOLINA EN EXPERIMENTO DE DICIEMBRE 22 DE 2017 


\section{Results and Discussion}

Successive values of $50 \mathrm{nA}, 320 \mu \mathrm{A}, 780 \mu \mathrm{A}, 2.7 \mathrm{~mA}$ and $4.8 \mathrm{~mA}$ under five different conditions were measured. Increasing values of apparent conductivities and decreasing values of resistivities were calculated according to the equations presented in table 1. Analysis of these results based in equations (6) and (7) revealed that correspond to increasing currents, ions increasing and conductivities increasing (resistivities decreasing) in the gasoline from a minimum (section enlargement) to a maximum (with minimizer installed) passing for magnets externally placed on the fuel supply hose and with placed within the fuel supply hose.

\section{Conclusions}

The increase of measured current through the multimeter resistance implies an increase of current through the gasoline (not measured) as is explained in the conclusions support. This also implies ions increase, conductivity increase (decrease of resistivity) and consequently a global ionization of the fuel. The highest value of current was obtained when before the magnetic field action there was a fuel conditioning by a section enlargement (with Minimizer installed). This preconditioning results in more efficient performance of the magnetic devices (minimizer in this case). The influence of the section enlargement in increasing the ionization of the gasoline is very important as can be evaluated from table 1 The magnetic field action on the fuel is more efficient when magnets are within the conduction hose than placed externally to it. This experiment is important not only to prove the cited conjectures but as a procedure that with some improvements could provide important information for the engineering design of a specific device.

\subsection{Conclusions Support}

The conjecture (main objective) that a magnetic action ionizes the fuel is equivalent to the creation of ions and/or increasing ions in the fuel. In basic classic electromagnetism, this has three important implications as follows:

- Resistivity decrease (Conductivity increase),

- Consequent resistance decrease of a fuel sample

- Current increase through the gasoline.

If the main conjecture is correct, the increasing currents with respect to the unaltered conditions could be measured in the fuel. The DC current through gasoline with the UT50A multimeter cannot be measured directly, nor by other more precise commercial multimeters because the magnitude order of that current is about $10^{-15} \mathrm{~A}$. This range of currents are usually measured by special methods as the normalized method employed by INTI (Argentina). However, we can prove all the proposed conjectures taking advantage of the high resistance of the gasoline sample that sets up a circuit of two resistances in parallel; considering its resistance and the multimeter inner resistance. We also take advantage of the known values of the conductivity (resistivity) of gasoline and the calculated current through gasoline and its resistance, for the case of unaltered conditions in gasoline. To perform the explanation we use the ideal circuit shown in Figure 1. If the main conjecture is correct, As shown in Equation (1), the total DC current should increase followed by $I_{J}$ and $i_{j}$. This is due to the changes in initial conditions in gasoline by magnetic action with or without pre-treatment that should create and/or increase ions in the whole circuit with respect to the unaltered conditions. $\left.I_{T_{j}}=I_{J}+i_{j} 1\right) \quad I_{T_{j}}=$ Total Current $I_{j}=$ Current in gasoline

$i_{J}=$ Current through the multimeter resistance $j=0,1,2,3,4, \ldots$

Initial conditions are: $\rho_{0}=10^{13} \Omega m ; R_{0}=3.1 \times 10^{15} \Omega ; I_{0}=3.8 \times 10^{-15} A ; i_{0}=5.0 \times 10^{-8} A ; \quad r_{0}=2.4 \times$ $10^{-8} \Omega$; Condition 1 Section enlargement with splitting: $\rho_{1}, R_{1}, i_{1}, r_{1}$; Condition 2 Only magnets outside on the fuel supply hose: $\rho_{2}, R_{2}, i_{2}, r_{2}$; Condition 3 Only magnets inside fuel supply hose: $\rho_{3}, R_{3}, i_{3}, r_{3}$; Condition 4 Section enlargement with splitting and magnetic action: $\rho_{4}, R_{4}, i_{4}, r_{4}$

Instead of resistivities, conductivities $\sigma_{j}$ could be used as well. $\left.I_{T}=\frac{V}{R_{e_{i}}} \quad 2\right) \quad i=0,1,2,3,4, \ldots$

$V=$ Potencial difference between 2 points in the gasoline $V=$ constant $=12$ volts

$R_{e_{i}}=$ Equivalent resistence of the circuit $\frac{1}{R_{e_{i}}}=\frac{1}{R_{j}}+\frac{1}{r_{j}} 3$ )

$R_{j}=$ Resistence in portion of gasoline

$r_{j}=$ Resistence in multimeter

$R_{j}=\frac{\rho_{j} l}{A}=k \rho_{j}$ 
Then, due to constant V and based on Equations (1), (2), (3), (4), (5), the resistances $R_{e_{i}}$ and $R_{j}$ should all decrease with respect to correspondent initial values. Although the real values of $R_{e_{i}}, R_{j}$ and $\rho_{j}$ cannot be measured, decreasing measured currents are coherent with all the hypothesis emerging from the main conjecture because we have: $I_{4}>I_{3}>$ $I_{2}>I_{1}>I_{0}$ 6) as a consequence of $i_{4}>i_{3}>i_{2}>i_{1}>i_{0}$ 7) $I_{j}=\frac{V}{R_{j}}$ 8), then: $\frac{V}{R_{4}}>\frac{V}{R_{3}}>\frac{V}{R_{2}}>\frac{V}{R_{1}}>\frac{V}{R_{0}} \quad 9$ ) replacing $R_{j}$ values and simplifying we get: $\left.\frac{1}{\rho_{4}}>\frac{1}{\rho_{3}}>\frac{1}{\rho_{2}}>\frac{1}{\rho_{1}}>\frac{1}{\rho_{0}} 10\right)$, then $\rho_{4}<\rho_{3}<\rho_{2}<\rho_{1}<\rho_{0}$ 11) these resistivities were calculated from values of measured current $i_{j}=\frac{I_{j} \rho_{j} l}{A r_{j}} \quad$ that are proportional to the correspondent values of current $I_{J}$ (they are apparent resistivities). These values could be useful to size the magnetic minimizer if they are related to the obtained gas emission results from an ADC. Only their relative values are determinant to prove the conjectures and achieve the proposed objectives. From Equation (10), once can obtain $\sigma_{4}>\sigma_{3}>\sigma_{2}>\sigma_{1}>\sigma_{0}$ (increasing conductivities in gasoline that is the inverse of resistivities in comparison to that of unaltered conditions). After checking with the results obtained from an ADC, the experimental set up could be patented and used formally to size the magnetic minimizer.

\section{Acknowledgments}

The authors would like to acknowledge the support of Professor Remberto Avila Castilla, acting head and responsible person for the physics laboratory of the faculty of mathematical and natural sciences of Universidad de Cartagena, Colombia, in the assembly of the experimental set up and measuring of experiment quantities; and Engineer Diego J. Petro Porto for his opinions about specific properties of gasoline. In addition, the authors would like to acknowledge the support of the University of Ryerson.

\section{References}

[1] ETHOS. [Online]. Available: https://www.environmental-expert.com/news/iranian-oil-magnate-cuts-emissionsusing-12-million-fuel-magnets-4369

[2] ETHOS. [Online]. Available: http://ethosplan.com/empsfuel.aspx

[3] ECONOPLUS, Argentina, 2004. [Online]. Available: http://www.fueloptimiser.com.ar

[4] EBAY USA, (February 13, 2018). [Online]. Available: http://www.ebay.com/bhp/magnetic-fuel-saver

[5] R. Guerrero and M. Mehrvar, "High Efficiency Device to Cut Gases Emissions from Mobile Sources" in RTESE'17, Toronto, Canada, 2017.

[6] R. Guerrero, "High Efficiency Device to Cut Gases Emissions from Mobile Sources," in RTESE'17, Toronto, Canada, 2017.

[7] INTI, Argentina Procedimiento Específico PEE53. [Online]. Available: https://www.inti.gob.ar/fisicaymetrologia/pdf/pce/pee53.pdf

[8] SOCIDOC.COM, (November 7, 2017), UT50A/B/C Manual. [Online]. Available: https://socidoc.com/download/ut50abc-eng-manual_5a01de4dd64ab2e82e187a4c_pdf

[9] R. Roberts, Roberts and Roberts, Using Properties to manage Flammable Liquid Hazards, (February 5, 2011) [Online]. Available: roberts.com/documents/Using\%20Properties\%20to\%20Manage\%20Flammable\%20Liquid\%20Hazards.pdf

[10] T. V. Rakesh, G. Venkateswara Rao, P. Kalyan Sankar, "Fuel Ionization By a Magnetic Field In a Four Stroke SI Engine," International Journal of Mechanical Engineering and Computer Applications, vol. 2, no. 3, 2015. [Online]. Available: http://www.ijmca.org/index.php/ojs/article/view/129/pdf

[12] USPTO USA, (February 12, 2018). [Online] Available: https://www.uspto.gov

[13] USPTO USA, (February 12, 2018). [Online] Available: http://patft.uspto.gov/netahtml/PTO/search-bool.html

[14] EPO, Europe, (February 12, 2018). [Online] Available: https://lp.espacenet.com 